\title{
Supporting Information for : Reversible Calcium Plating and Stripping at Room Temperature Using a Borate Salt
}

\author{
Abhinandan Shyamsunder ${ }^{a}$, Lauren E. Blanc ${ }^{a}$, Abdeljalil Assoud ${ }^{a}$, Linda F. Nazar ${ }^{a^{\prime} *}$ \\ a Department of Chemistry and Waterloo Institute of Nanotechnology, University of Waterloo, \\ 200 University Avenue West, Waterloo, Ontario, N2L3G1, Canada. \\ 'Joint Center for Energy Storage Research, Argonne National Laboratory, Lemont, Illinois \\ 60439, United States
}

\section{Contents :}

SI.1 Experimental Details

SI.2 Synthetic Procedures

SI.3 List of Figures

SI.4 Single crystal data 


\section{SI.1 Experimental details:}

All reactions were carried out under a dry, oxygen free argon atmosphere using standard Schlenk techniques or in a glovebox with $\mathrm{O}_{2}$ and $\mathrm{H}_{2} \mathrm{O}$ levels less than $5 \mathrm{ppm}$. Calcium metal (99\%), borane dimethylsulfide complex in pentane, calcium methoxide $(95 \%)$, tetrabutyl ammonium chloride $(97 \%)$ were purchased from Sigma Aldrich and were used as received. The solvent 1,2-dimethoxy ethane (Sigma Aldrich) was passed over a column of activated alumina, distilled over Na, degassed and stored over molecular sieves $\left(\mathrm{H}_{2} \mathrm{O}<1 \mathrm{ppm}\right.$, undetectable by Karl-Fischer $)$ inside the box. Hexafluoroisopropanol (99\%) was purchased from Oakwood Chemicals and was distilled over calcium hydride, stored under $3 \AA$ molecular sieves and was distilled over $\mathrm{CaH}_{2}$ again before use. Tetrahydrofuran (Sigma Aldrich) was predried with $\mathrm{KOH}$, and distilled over $\mathrm{Na}$ and benzophenone, degassed and stored over molecular sieves in an amber bottle inside the glovebox.

NMR spectra was obtained on a Bruker Avance II spectrometer at $7.05 \mathrm{~T}$ for the ${ }^{1} \mathrm{H},{ }^{19} \mathrm{~F}$ and ${ }^{11} \mathrm{~B}$ nuclei. ${ }^{1} \mathrm{H}$ spectra were recorded $300 \mathrm{MHz}$ and the peaks are referenced to the residual $\mathrm{CH}_{3} \mathrm{CN}$ peak $(2.1 \mathrm{ppm}) ;{ }^{19} \mathrm{~F}$ spectra were recorded at $282.231 \mathrm{MHz}$ and are referenced to the trifluoroacetic acid (-76.5 ppm); ${ }^{11} \mathrm{~B}$ spectra were recorded at $96.251 \mathrm{MHz}$ and are referenced to boric acid. All samples were prepared using $\mathrm{CD}_{3} \mathrm{CN}$ (Sigma Aldrich) as the solvent.

Air and moisture sensitive crystals of $\mathrm{Ca}\left[\mathrm{C}_{2} \mathrm{H}_{4}\left(\mathrm{OCH}_{3}\right)_{2}\right]_{4} \cdot 2 \mathrm{~B}\left[\mathrm{OCH}\left(\mathrm{CF}_{3}\right)_{2}\right]_{4}$ were prepared in the glovebox and protected using Paratone oil. The colourless block like crystals were selected under an optical microscope and mounted on glass fibres, then protected with a $\mathrm{N}_{2}$ flow using OXFORD Cryostream 700 and an AD51 Dry air to cool the crystals to the desired temperature. Two data sets at 280 and $200 \mathrm{~K}$ were collected using a Bruker Kappa Apex II equipped with a Charge Coupled Device detector utilizing graphite monochromatic Mo-K $\alpha$ radiation $(\lambda=0.71073 \AA)$. A complete data was collected by scanning $\omega$ in different regions using the APEX3 search strategy, with exposure time of 40 seconds per frame. The data were corrected for Lorentz and polarization effects using APEX3 Suite Software, and the absorption corrections were fitted empirically using SADABS. The structure solutions were obtained by direct method and refined using the least square method incorporated into the SHELXTL package. All the non-hydrogen atoms were refined anisotropically and all the hydrogen atoms were refined in the idealized geometrically positions using a rigid model with $\mathrm{C}-\mathrm{H}=0.93 \AA$ and isotropic displacement parameters $\mathrm{U}_{\text {iso }}(\mathrm{H})=1.2 \mathrm{U}_{\text {eq }}(\mathrm{C})$ and $\mathrm{U}_{\text {iso }}(\mathrm{H})=1.5 \mathrm{U}_{\text {eq }}(\mathrm{C})$ for the $\mathrm{CH}_{3}$ group. Both models show disorder of the coordinated solvent molecule with the $280 \mathrm{~K}$ model being the most pronounced. The crystallographic data, atomic coordinates, atomic thermal displacement parameters and bond distances and angles of the $200 \mathrm{~K}$ data are regrouped in Tables S1-S4.

Electrochemical studies were carried out in three electrode Swagelok ${ }^{\mathrm{TM}}$ T-cells, using Au (10 mm disks) as the working electrode, and calcium metal as the reference and counter electrodes (12 mm diameter). Celgard ${ }^{\mathrm{TM}}$ and glass fiber separators were used with a volume of $150 \mu \mathrm{L}$ of $0.5 \mathrm{M}$ $\mathrm{Ca}\left(\mathrm{B}(\mathrm{Ohfip})_{4}\right)_{2}$ in $\mathrm{DME}$ electrolyte with or without $\mathrm{Bu}_{4} \mathrm{NCl}$. The $\mathrm{Au}$ disks were cleaned according to previously reported methods. ${ }^{1}$ Cyclic voltammetry (CV), linear sweep voltammetry (LSV), galvanostatic stripping and plating studies were performed with a VMP3 potentiostat (Bio-logic) at room temperature. 
Ionic conductivities were measured using a 3-star conductimeter (Orion) equipped with a two electrode epoxy conductivity probe (cell constant $1.0 \mathrm{~cm}^{-1}$ ).

Synchrotron XRD experiments were carried out at the Canadian Light Source (CLS) facility using a wavelength of $0.68926 \AA$. The data were fit by the Le Bail method using the FullProf suite to identify phases present in the deposited material.

Imaging was performed using a LEO 1530 Zeiss field emission scanning electron microscope (SEM) equipped with an energy dispersive X-ray spectrometer (EDX). Samples for SEM were obtained by disassembling the cells, washing the electrode thoroughly with THF and drying it under vacuum to ensure complete removal of residual electrolyte.

\section{SI.2 Synthetic Procedures}

\section{Calcium hexafluoroisopropoxide (Ca(Ohfip)2.xTHF) :}

Calcium methoxide (1 eq) was allowed to stir in THF for 2 hours at room temperature under argon. To the obtained dispersion was added $2.5 \mathrm{eq}$ of hexafluoroisporopanol and the mixture was refluxed for 4 days resulting in a slightly cloudy solution. The solution was filtered, and the clear filtrate was concentrated under vacuum at $50{ }^{\circ} \mathrm{C}$ to obtain a white solid, which was further dried at $90{ }^{\circ} \mathrm{C}$ for two hours under dynamic vacuum affording a white powder. ${ }^{1} \mathrm{H}$ NMR $(300 \mathrm{MHz}$, $\left.\mathrm{CD}_{3} \mathrm{CN}\right): \delta=4.71(\mathrm{~m}), 3.75(\mathrm{t}), 1.85(\mathrm{t}) .{ }^{19} \mathrm{~F} \mathrm{NMR}\left(282 \mathrm{MHz} \mathrm{CD}_{3} \mathrm{CN}\right): \delta=-77.2$.

\section{Tris-hexafluoroisopropoxy borate (B(Ohfip)3):}

Tris-hexafluoroisopropoxy borate was prepared according to a previously reported procedure ${ }^{2}$ and chemical analysis was in agreement with the expected composition. This borate salt was sublimed before use.

\section{Calcium bis(hexafluroisopropoxy borate) ( $\left.\mathrm{Ca}(\mathrm{B}(\mathrm{Ohfip}) 4)_{2.4 D M E}\right)$ :}

Calcium hexafluoroisopropoxide ( $\mathrm{Ca}$ (Ohfip) 2.xTHF) and tris-hexafluoroisopropoxy borate $\left(\mathrm{B}(\mathrm{Ohfip})_{3}\right)$ were mixed in appropriate ratios in 1,2-dimethoxyethane (DME) at $65^{\circ} \mathrm{C}$ for 6 hours. The clear solution obtained was then concentrated under dynamic vacuum at room temperature to obtain the $\mathrm{Ca}(\mathrm{B} \text { (Ohfip) })_{4}$ 2.xDME salt. The obtained salt was redissolved in DME and subjected to a slow evaporation to obtain crystals of $\left.\mathrm{Ca}(\mathrm{B}(\mathrm{Ohfip}))_{4}\right)_{2} .4 \mathrm{DME} .{ }^{1} \mathrm{H} \mathrm{NMR}\left(300 \mathrm{MHz}, \mathrm{CD}_{3} \mathrm{CN}\right): \delta=$ 4.76(m), 3.55(t), 3.42(s). ${ }^{19} \mathrm{~F}$ NMR (282 $\left.\mathrm{MHz} \mathrm{CD}_{3} \mathrm{CN}\right): \delta=-75.8 .{ }^{11} \mathrm{~B} \mathrm{NMR}\left(96.25 \mathrm{MHz}, \mathrm{CD}_{3} \mathrm{CN}\right)$ : $\delta=9.2(\mathrm{~s})$. 

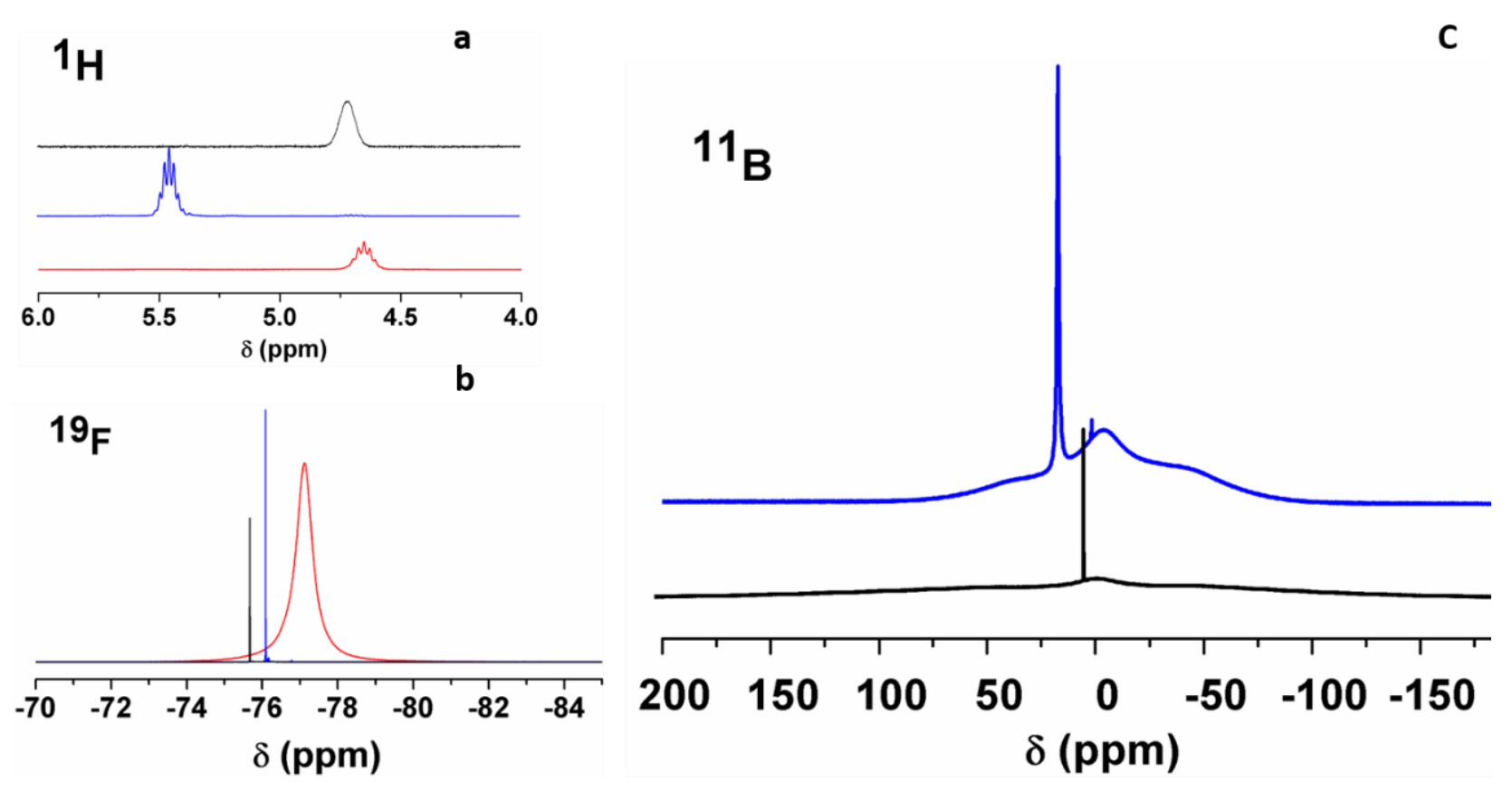

Figure S1. NMR spectra of the precursors and Ca salt product showing the a) ${ }^{1} \mathrm{H}$; b) ${ }^{19} \mathrm{~F}$; and c) ${ }^{11} \mathrm{~B}$ NMR spectra. Blue curves represent the boron ester, red curves represent the calcium alkoxide and the black curves correspond to the synthesized $\mathrm{Ca}\left(\mathrm{B}(\mathrm{Ohfip})_{4}\right)_{2} \bullet(\mathrm{DME})_{4}$ salt. Solvent peaks are omitted for clarity. 


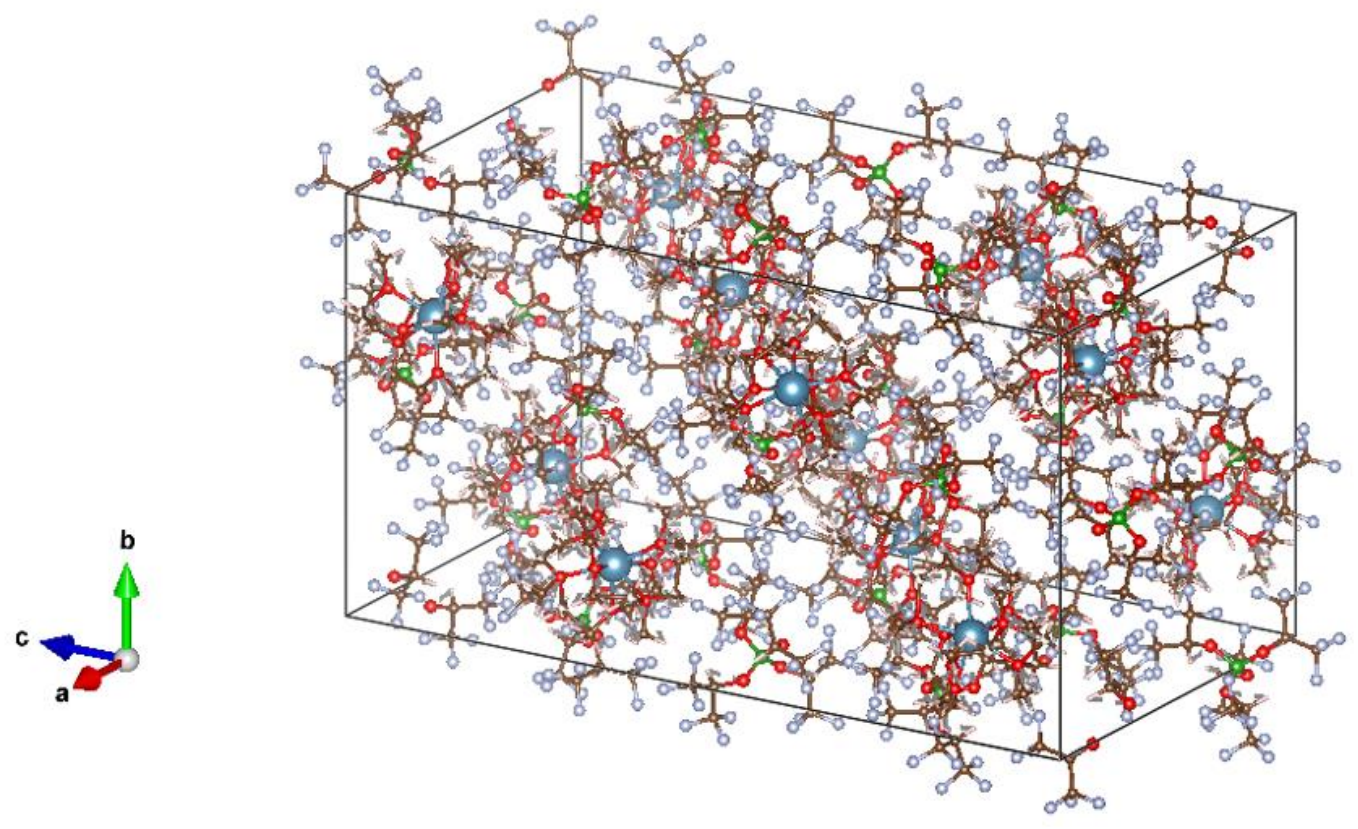

Figure S2. Refined unit cell of $\mathrm{Ca}\left(\mathrm{B}(\mathrm{Ohfip})_{4}\right)_{2} \bullet(\mathrm{DME})_{4}$ from single crystal analysis. Calcium (blue) is chelated to eight oxygens (red) of the four DME molecules and the boron (green) is attached to four oxygens from the hexafluoroisopropoxy ligands.
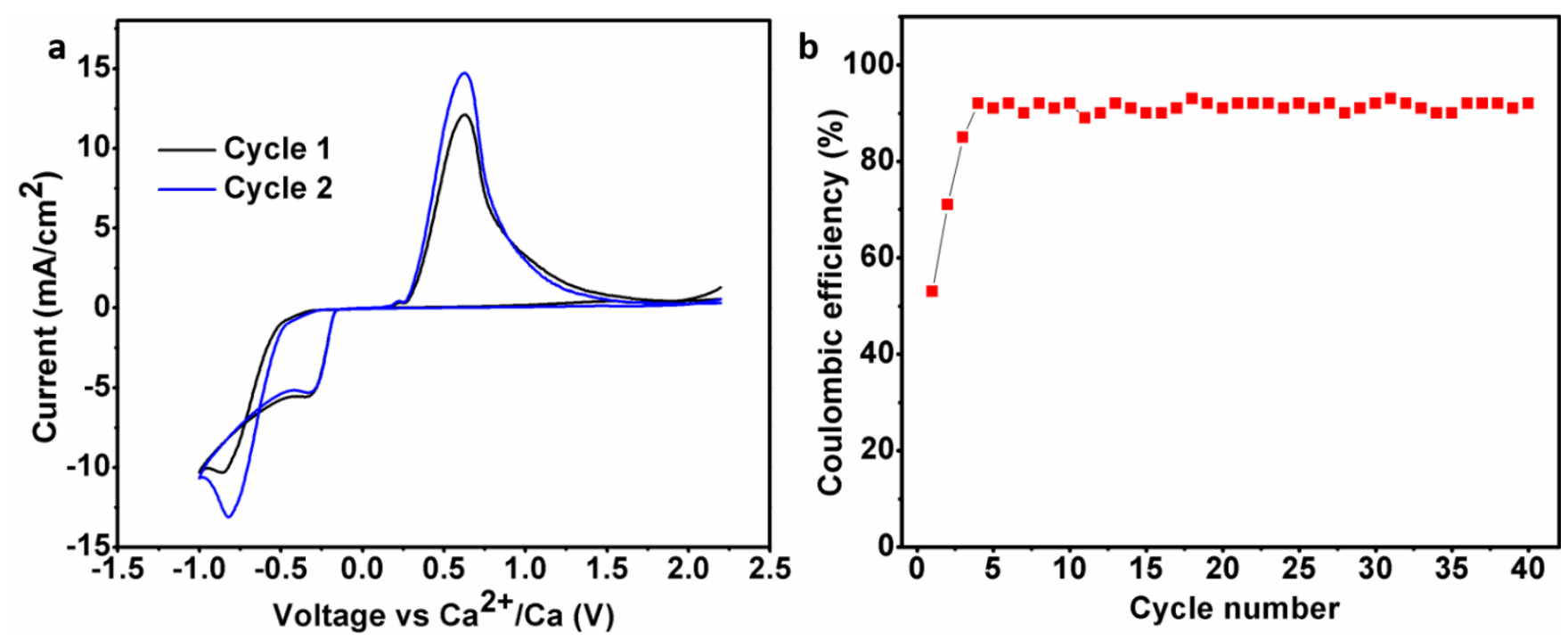

Figure S3. a) $1^{\text {st }}$ (black) and $2^{\text {nd }}$ (blue) CV cycle and b) plot of Coulombic efficiency $v s$ cycle numbers obtained from the $\mathrm{CV}$ experiment with a $\left.500 \mathrm{mM} \mathrm{Ca(B(Ohfip)})_{4}\right)_{2}$ electrolyte on an Au substrate with calcium as reference and counter electrodes at a scan rate of $25 \mathrm{mV} \cdot \mathrm{s}^{-1}$. 

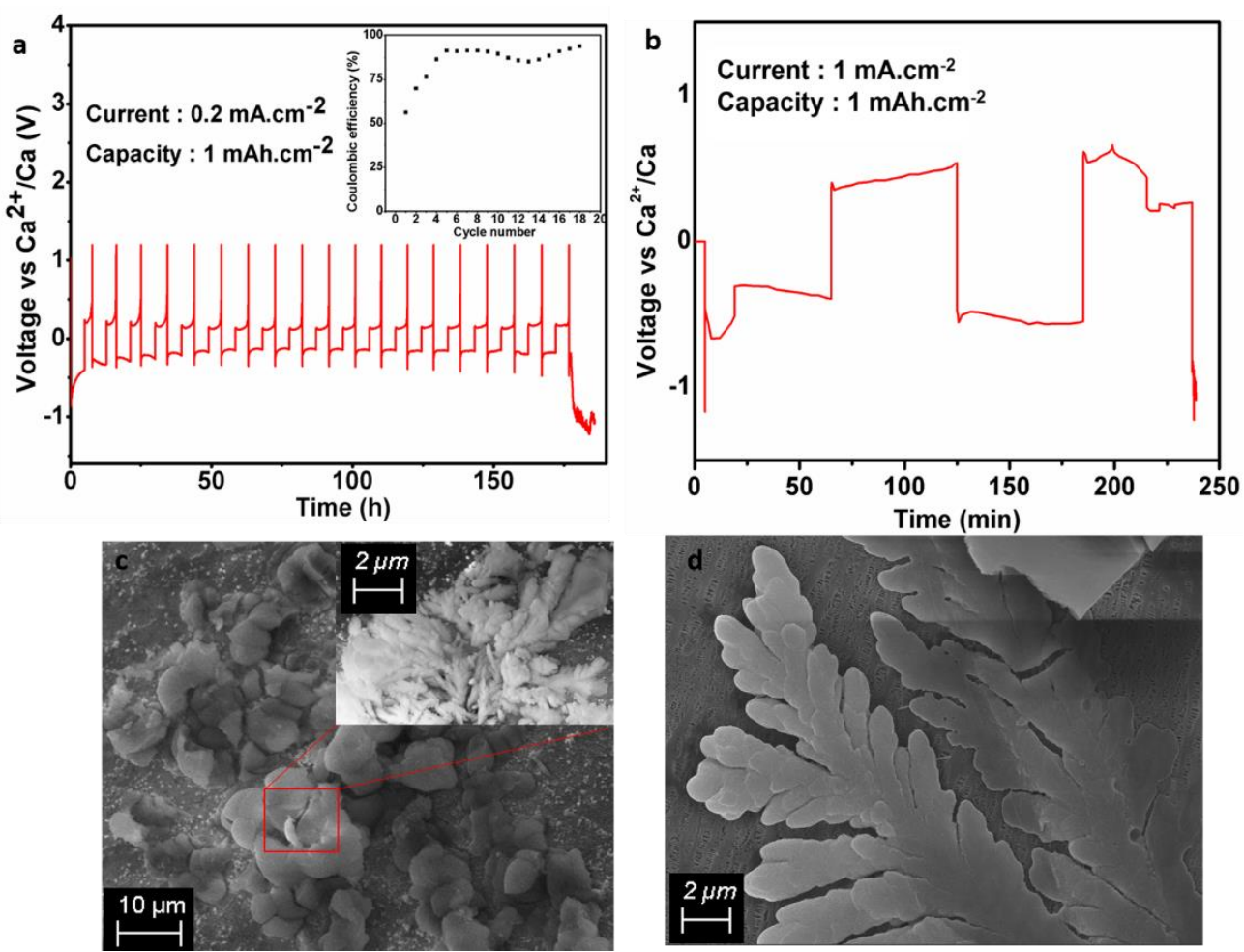

Figure S4. Galvanostatic stripping and plating calcium to a capacity of $1 \mathrm{mAh} \cdot \mathrm{cm}^{-2}$ with a) $0.2 \mathrm{~mA} \cdot \mathrm{cm}^{-2}$ current density and b) $1 \mathrm{~mA} \cdot \mathrm{cm}^{-2}$ current density with the inset in a) showing the coulombic efficiency. c) SEM image depicting non-uniform and patchy deposition of calcium with the inset showing a magnified image of the selected region; d) SEM image showing the dendritic calcium growing through the Celgard ${ }^{\mathrm{TM}}$ separator. EDX spectra (not shown) reveal the presence of fluorine in/on the dendrites (Ca: $\mathrm{F}$ of 1:1). The characteristic morphology of the dendrites suggests a diffusion limited aggregation (DLA) process occurs. 


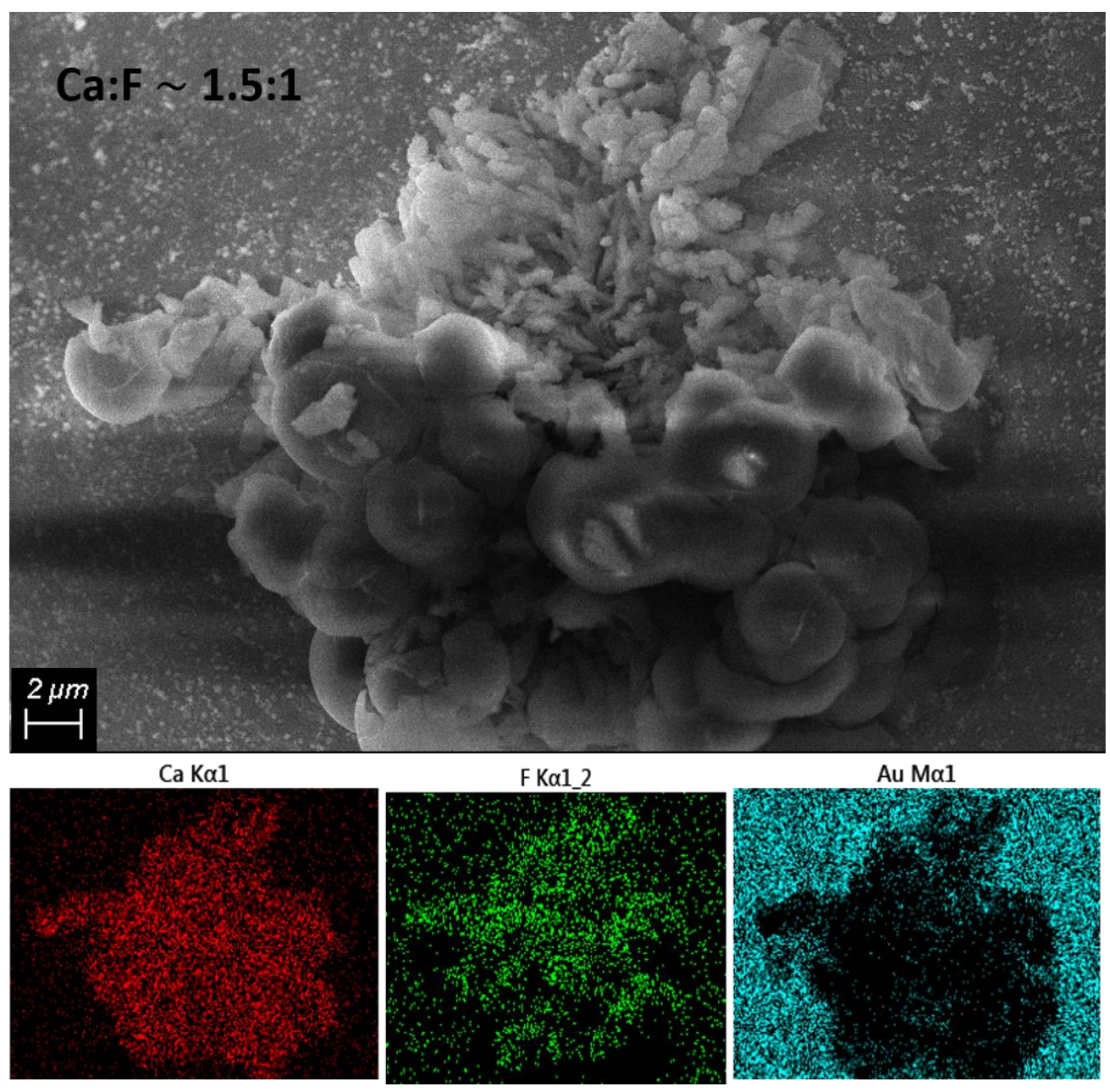

Figure S5. SEM image of an island deposit of calcium obtained from a cell that was galvanostatically discharged at $1 \mathrm{~mA} \cdot \mathrm{cm}^{-2}$ to $1 \mathrm{mAh} \cdot \mathrm{cm}^{-2}$ and the corresponding EDX elemental maps. 


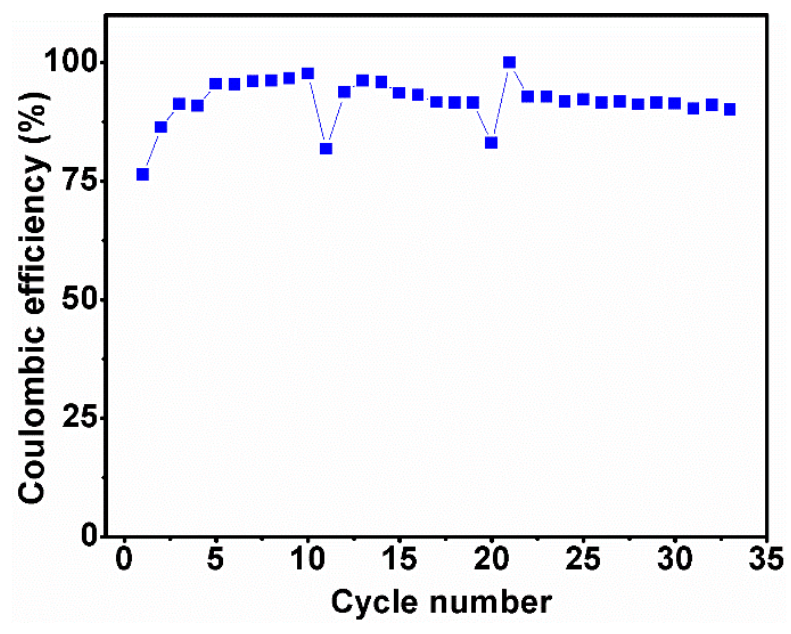

Figure S6. Plot of coulombic efficiency $v s$ cycle number for the galvanostatic stripping and plating of calcium at a current density of $0.5 \mathrm{~mA} \cdot \mathrm{cm}^{-2}$ in a three electrode cell using $\mathrm{Ca}\left(\mathrm{B}(\mathrm{Ohfip})_{4}\right)_{2} .4 \mathrm{DME}$ salt in DME +100 $\mathrm{mM} \mathrm{Bu}{ }_{4} \mathrm{NCl}$ as the electrolyte. Au served as the working electrode and $\mathrm{Ca}$ for the counter and reference electrodes.

a

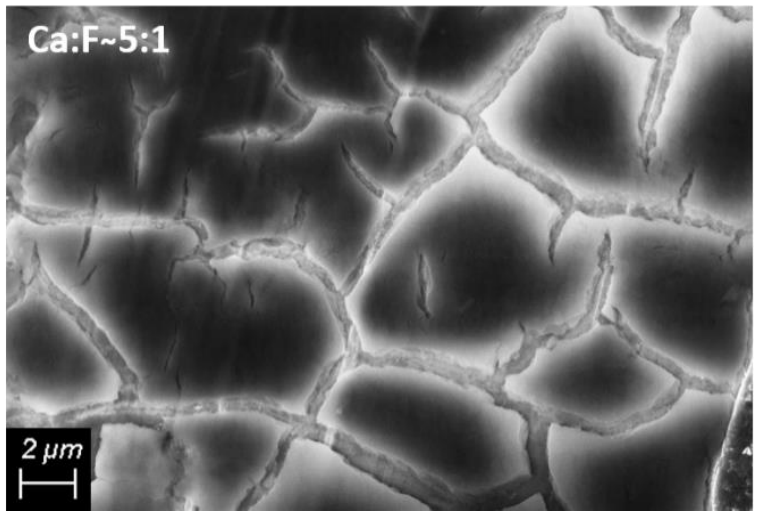

b

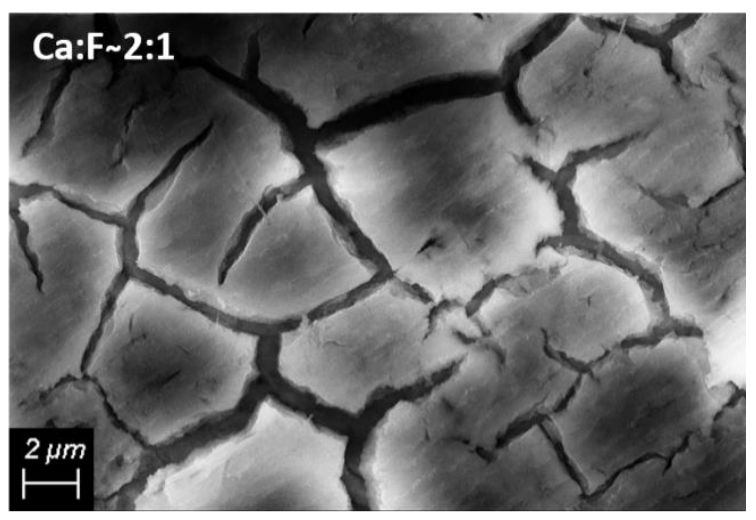

Figure S7. SEM image of a) $\mathrm{Cu}$ and b) SS electrode obtained from a cell galvanostatically discharged to $6 \mathrm{mAh} . \mathrm{cm}^{-2}$ using a $500 \mathrm{mM} \mathrm{Ca}\left(\mathrm{B}(\text { Ohfip })_{4}\right)_{2}$ and $100 \mathrm{mM} \mathrm{NBu} 4 \mathrm{Cl}$ electrolyte showing the island-like deposits that are similar to those on a gold electrode. 


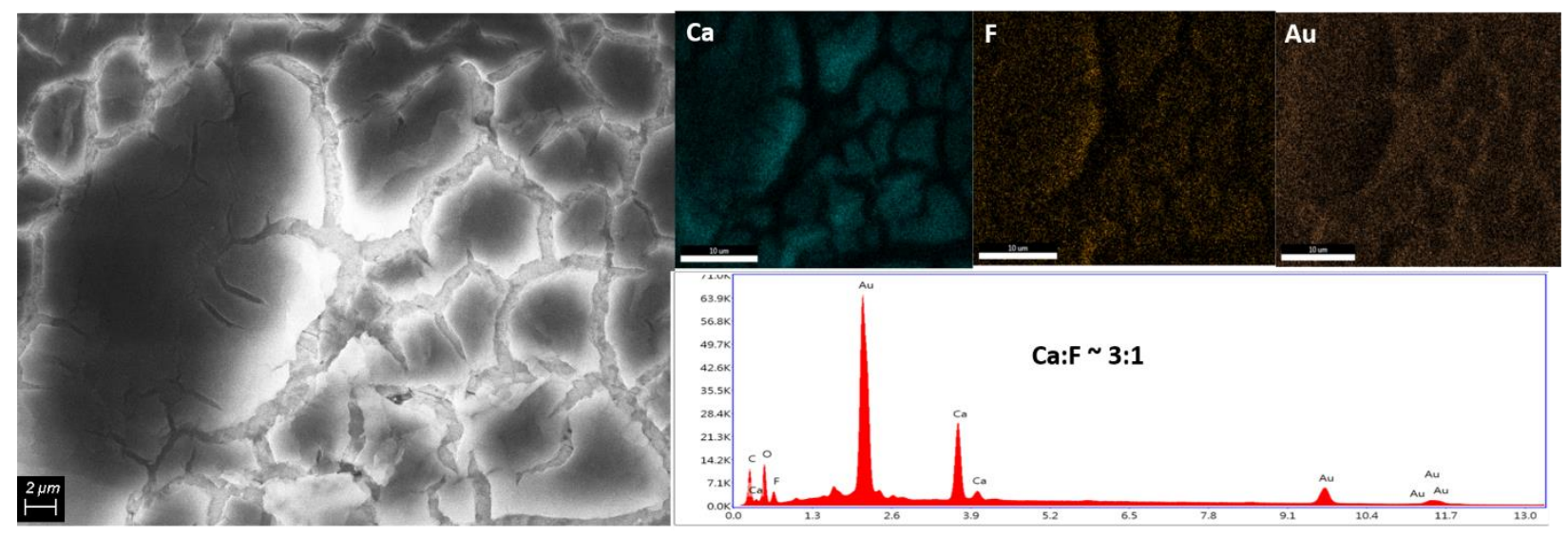

Figure S8. SEM image of the gold electrode obtained from a cell galvanostatically discharged to $6 \mathrm{mAh} . \mathrm{cm}^{-2}$ using a $500 \mathrm{mM} \mathrm{Ca}\left(\mathrm{B}(\text { Ohfip) })_{4}\right)_{2}+100 \mathrm{mM} \mathrm{NBu}_{4} \mathrm{Cl}$ electrolyte showing the nonuniform island like deposits of calcium and the corresponding elemental and EDX maps (white scale bar $=10 \mu \mathrm{m}$ ) showing the presence of calcium fluoride on the surface of the deposits.
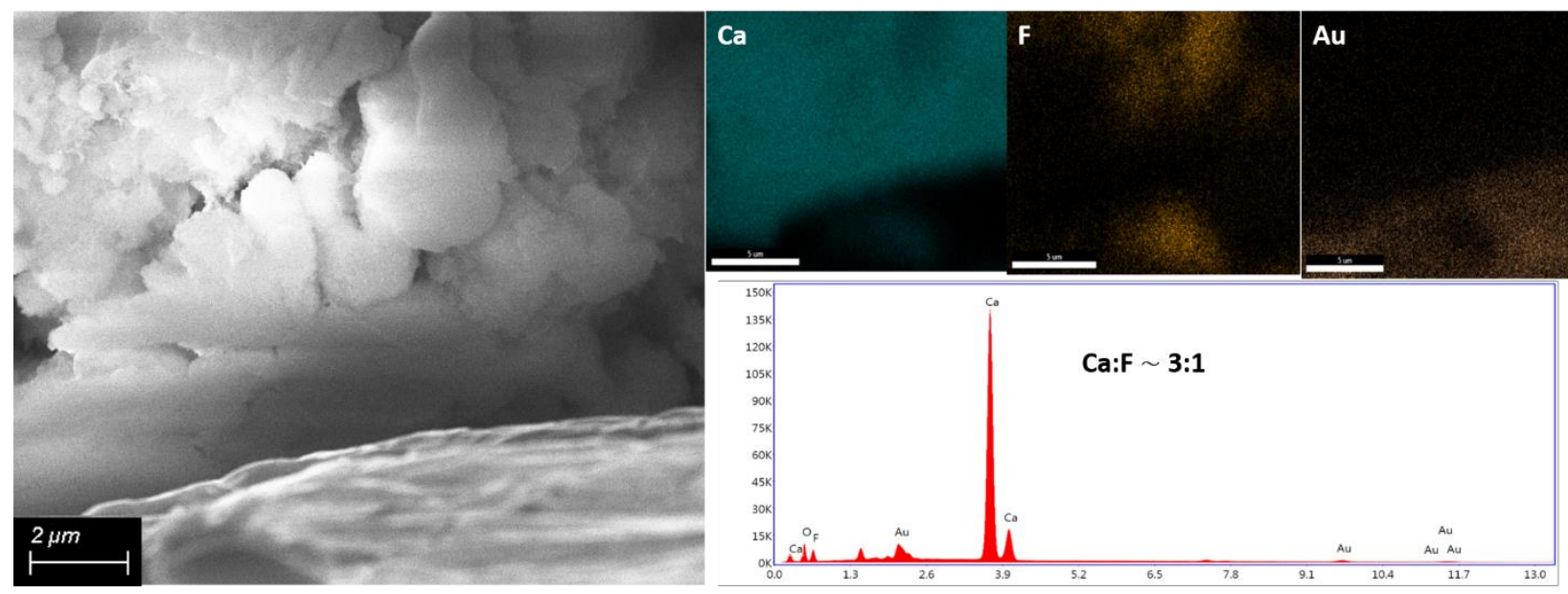

Figure S9. Cross sectional image of the gold electrode obtained from a cell galvanostatically discharged to $6 \mathrm{mAh} . \mathrm{cm}^{-2}$ using a $\left.\left.500 \mathrm{mM} \mathrm{Ca(B(Ohfip)}\right)_{4}\right)_{2}$ electrolyte and the associated elemental maps (white scale bar $=5 \mu \mathrm{m}$ ) indicating that the deposited product is predominantly calcium. 


\section{SI.4 Single crystal data:}

Table S1. Crystal data and structure refinement for $\mathrm{Ca}_{2}\left[\mathrm{C}_{2} \mathrm{H}_{4}\left(\mathrm{OCH}_{3}\right)_{2}\right]_{4} \bullet 2 \mathrm{~B}\left[\mathrm{OCH}\left(\mathrm{CF}_{3}\right)_{2}\right]_{4}$

\begin{tabular}{|c|c|}
\hline Empirical formula & $\mathrm{C}_{40} \mathrm{H}_{48} \mathrm{O}_{16} \mathrm{~F}_{48} \mathrm{~B}_{2} \mathrm{Ca}$ \\
\hline Formula weight & 1758.48 \\
\hline Temperature & $200(2) \mathrm{K}$ \\
\hline Wavelength & $0.71073 \AA$ \\
\hline Crystal system & Tetragonal \\
\hline Space group & $I 4_{1} /$ acd \\
\hline Unit cell dimensions & $a=b=19.6322(8) \AA, c=36.8549(15) \AA$ \\
\hline Unit cell angles & $\alpha=\beta=\gamma=90^{\circ}$ \\
\hline Volume & $14204.7(13) \AA^{3}$ \\
\hline $\mathrm{Z}$ & 8 \\
\hline Density (calculated) & $1.645 \mathrm{~g} / \mathrm{cm}^{3}$ \\
\hline Absorption coefficient & $0.269 \mathrm{~mm}^{-1}$ \\
\hline $\mathrm{F}(000)$ & 7024 \\
\hline Crystal size & $0.160 \times 0.140 \times 0.080 \mathrm{~mm}^{3}$ \\
\hline Theta range for data collection & 2.210 to $25.996^{\circ}$. \\
\hline Index ranges & $-23<=\mathrm{h}<=24,-18<=\mathrm{k}<=24,-44<=1<=45$ \\
\hline Reflections collected & 59346 \\
\hline Independent reflections & $3501[\mathrm{R}(\mathrm{int})=0.0829]$ \\
\hline Completeness to theta $=25.242^{\circ}$ & $99.9 \%$ \\
\hline Absorption correction & Semi-empirical from equivalents \\
\hline Max. and min. transmission & 0.7460 and 0.6992 \\
\hline Refinement method & Full-matrix least-squares on $\mathrm{F}^{2}$ \\
\hline Data / restraints / parameters & $3501 / 0 / 250$ \\
\hline Goodness-of-fit on $\mathrm{F}^{2}$ & 1.182 \\
\hline Final $\mathrm{R}$ indices $[\mathrm{I}>2 \operatorname{sigma}(\mathrm{I})]$ & $\mathrm{R} 1=0.0737, \mathrm{wR} 2=0.1312$ \\
\hline $\mathrm{R}$ indices (all data) & $\mathrm{R} 1=0.1291, \mathrm{wR} 2=0.1543$ \\
\hline Extinction coefficient & $\mathrm{n} / \mathrm{a}$ \\
\hline Largest diff. peak and hole & 0.720 and -0.330 e. $\AA^{-3}$ \\
\hline
\end{tabular}


Table S2. Atomic coordinates $\left(\times \mathbf{1 0}^{4}\right)$ and equivalent isotropic displacement parameters $\left(\AA^{2} \times 10^{3}\right)$ for $\mathrm{C}_{40} \mathrm{H}_{48} \mathrm{O}_{16} \mathrm{~F}_{48} \mathrm{~B}_{2} \mathrm{Ca}$ at $200 \mathrm{~K}$

\begin{tabular}{|c|c|c|c|c|}
\hline & $\mathrm{X}$ & $\mathrm{y}$ & $\mathrm{Z}$ & $\mathrm{U}_{\mathrm{eq}}$ \\
\hline $\mathrm{B}(1 \mathrm{~A})$ & 2500 & $4990(4)$ & 5000 & $59(2)$ \\
\hline $\mathrm{C}(1 \mathrm{~A})$ & $1735(3)$ & $4381(2)$ & $4566(1)$ & $73(1)$ \\
\hline $\mathrm{C}(2 \mathrm{~A})$ & $1643(4)$ & $4561(4)$ & $4172(2)$ & $97(2)$ \\
\hline$C(3 \mathrm{~A})$ & $1628(4)$ & $3623(3)$ & $4636(2)$ & $109(2)$ \\
\hline $\mathrm{C}(4 \mathrm{~A})$ & $3318(3)$ & $5603(2)$ & $4600(1)$ & $67(1)$ \\
\hline$C(5 A)$ & $4045(3)$ & $5374(3)$ & $4540(2)$ & $93(2)$ \\
\hline$C(6 A)$ & $3226(4)$ & $6371(3)$ & $4560(2)$ & $88(2)$ \\
\hline $\mathrm{O}(1 \mathrm{~A})$ & $2383(2)$ & $4575(1)$ & $4672(1)$ & $63(1)$ \\
\hline $\mathrm{O}(2 \mathrm{~A})$ & $3113(2)$ & $5397(1)$ & $4942(1)$ & $61(1)$ \\
\hline $\mathrm{F}(1 \mathrm{~A})$ & $1012(2)$ & $4443(2)$ & $4057(1)$ & $139(2)$ \\
\hline $\mathrm{F}(2 \mathrm{~A})$ & $2067(2)$ & $4254(2)$ & $3952(1)$ & $139(2)$ \\
\hline $\mathrm{F}(3 \mathrm{~A})$ & $1759(2)$ & $5226(2)$ & $4128(1)$ & $115(1)$ \\
\hline $\mathrm{F}(4 \mathrm{~A})$ & $1010(2)$ & $3415(2)$ & $4535(1)$ & $153(2)$ \\
\hline $\mathrm{F}(5 \mathrm{~A})$ & $2073(2)$ & $3239(2)$ & $4467(1)$ & $150(2)$ \\
\hline $\mathrm{F}(6 \mathrm{~A})$ & $1682(2)$ & $3485(2)$ & 4989(1) & $139(2)$ \\
\hline $\mathrm{F}(7 \mathrm{~A})$ & $4480(2)$ & $5639(2)$ & $4771(1)$ & $125(1)$ \\
\hline $\mathrm{F}(8 \mathrm{~A})$ & $4089(2)$ & $4703(2)$ & $4586(1)$ & $136(2)$ \\
\hline $\mathrm{F}(9 \mathrm{~A})$ & $4271(2)$ & $5510(2)$ & $4209(1)$ & $134(1)$ \\
\hline $\mathrm{F}(10 \mathrm{~A})$ & $3452(2)$ & $6597(2)$ & $4243(1)$ & $132(1)$ \\
\hline $\mathrm{F}(11 \mathrm{~A})$ & $3563(2)$ & $6712(2)$ & $4814(1)$ & 111(1) \\
\hline $\mathrm{F}(12 \mathrm{~A})$ & 2582(2) & $6539(2)$ & $4586(1)$ & $122(1)$ \\
\hline
\end{tabular}




\begin{tabular}{|c|c|c|c|c|}
\hline & $\mathrm{x}$ & $\mathrm{y}$ & $\mathrm{z}$ & $\mathrm{U}_{\mathrm{eq}}$ \\
\hline $\mathrm{Ca}(1)$ & 0 & 7500 & 3750 & $47(1)$ \\
\hline $\mathrm{O}(1 \mathrm{~B})$ & 1201(2) & 7796(3) & $3881(1)$ & $130(2)$ \\
\hline$C(1 B)$ & 1403(4) & $8496(4)$ & $3879(3)$ & $179(4)$ \\
\hline$C(2 B)$ & $1692(4)$ & $7350(5)$ & $4030(3)$ & $226(6)$ \\
\hline $\mathrm{O}(2 \mathrm{~B})$ & $-319(3)$ & $8084(4)$ & $4307(2)$ & $186(3)$ \\
\hline$C(3 B)$ & $-492(5)$ & $8808(5)$ & $4327(3)$ & $215(5)$ \\
\hline$C(4 B)$ & $-76(12)$ & 7873(13) & $4639(3)$ & $360(20)$ \\
\hline$C(5 B)$ & $1190(30)$ & $7280(40)$ & $4391(8)$ & $360(20)$ \\
\hline $\mathrm{H}(1 \mathrm{~A})$ & 1395 & 4643 & 4712 & 88 \\
\hline $\mathrm{H}(4 \mathrm{~A})$ & 3022 & 5372 & 4417 & 80 \\
\hline $\mathrm{H}(1 \mathrm{BA})$ & 1891 & 8528 & 3813 & 215 \\
\hline $\mathrm{H}(1 \mathrm{BB})$ & 1349 & 8687 & 4126 & 215 \\
\hline $\mathrm{H}(2 \mathrm{BA})$ & 1994 & 7606 & 4192 & 339 \\
\hline $\mathrm{H}(2 \mathrm{BB})$ & 1961 & 7147 & 3834 & 339 \\
\hline $\mathrm{H}(2 \mathrm{BC})$ & 1462 & 6990 & 4167 & 339 \\
\hline $\mathrm{H}(3 \mathrm{BA})$ & -233 & 9022 & 4524 & 322 \\
\hline $\mathrm{H}(3 \mathrm{BB})$ & -980 & 8858 & 4374 & 322 \\
\hline $\mathrm{H}(3 \mathrm{BC})$ & -377 & 9029 & 4096 & 322 \\
\hline $\mathrm{H}(4 \mathrm{BA})$ & -419 & 7973 & 4829 & 434 \\
\hline $\mathrm{H}(4 \mathrm{BB})$ & 344 & 8129 & 4699 & 434 \\
\hline $\mathrm{H}(5 \mathrm{BA})$ & 1141 & 7734 & 4502 & 434 \\
\hline $\mathrm{H}(5 \mathrm{BB})$ & 1428 & 6985 & 4571 & 434 \\
\hline
\end{tabular}

$\mathrm{U}(\mathrm{eq})$ is defined as one third of the trace of the orthogonalized $\mathrm{U}_{\mathrm{ij}}$ tensor. 
Table S3. Anisotropic displacement parameters $\left(\AA^{2} x 1^{3}\right)$ for $\mathrm{C}_{40} \mathrm{H}_{48} \mathrm{O}_{16} \mathrm{~F}_{48} \mathrm{~B}_{2} \mathrm{Ca}$ at $200 \mathrm{~K}$

\begin{tabular}{|l|l|l|l|l|l|l|}
\hline & $\mathrm{U}_{11}$ & $\mathrm{U}_{22}$ & $\mathrm{U}_{33}$ & $\mathrm{U}_{23}$ & $\mathrm{U}_{13}$ & $\mathrm{U}_{12}$ \\
\hline $\mathrm{B}(1 \mathrm{~A})$ & $83(5)$ & $46(4)$ & $49(3)$ & 0 & $-6(4)$ & 0 \\
\hline $\mathrm{C}(1 \mathrm{~A})$ & $83(4)$ & $68(3)$ & $70(3)$ & $-18(3)$ & $-2(3)$ & $-11(3)$ \\
\hline $\mathrm{C}(2 \mathrm{~A})$ & $103(5)$ & $108(5)$ & $80(4)$ & $-27(4)$ & $-19(4)$ & $1(4)$ \\
\hline $\mathrm{C}(3 \mathrm{~A})$ & $118(6)$ & $82(5)$ & $127(6)$ & $-22(4)$ & $-7(5)$ & $-29(4)$ \\
\hline $\mathrm{C}(4 \mathrm{~A})$ & $82(4)$ & $66(3)$ & $53(3)$ & $2(2)$ & $-9(2)$ & $-15(3)$ \\
\hline $\mathrm{C}(5 \mathrm{~A})$ & $106(5)$ & $96(5)$ & $77(4)$ & $-8(4)$ & $10(4)$ & $-6(4)$ \\
\hline $\mathrm{C}(6 \mathrm{~A})$ & $116(5)$ & $73(4)$ & $74(4)$ & $19(3)$ & $-13(4)$ & $-20(4)$ \\
\hline $\mathrm{O}(1 \mathrm{~A})$ & $77(2)$ & $54(2)$ & $59(2)$ & $-10(1)$ & $-2(2)$ & $-6(2)$ \\
\hline $\mathrm{O}(2 \mathrm{~A})$ & $79(2)$ & $54(2)$ & $49(2)$ & $3(1)$ & $-7(2)$ & $-7(2)$ \\
\hline $\mathrm{F}(1 \mathrm{~A})$ & $115(3)$ & $190(4)$ & $112(3)$ & $-32(3)$ & $-43(2)$ & $-11(3)$ \\
\hline $\mathrm{F}(2 \mathrm{~A})$ & $153(3)$ & $184(4)$ & $79(2)$ & $-47(2)$ & $1(2)$ & $25(3)$ \\
\hline $\mathrm{F}(3 \mathrm{~A})$ & $134(3)$ & $127(3)$ & $85(2)$ & $18(2)$ & $-24(2)$ & $1(2)$ \\
\hline $\mathrm{F}(4 \mathrm{~A})$ & $130(3)$ & $122(3)$ & $207(4)$ & $-27(3)$ & $-22(3)$ & $-58(3)$ \\
\hline $\mathrm{F}(5 \mathrm{~A})$ & $171(4)$ & $69(2)$ & $209(5)$ & $-47(3)$ & $21(3)$ & $3(2)$ \\
\hline $\mathrm{F}(6 \mathrm{~A})$ & $172(4)$ & $94(3)$ & $151(3)$ & $28(3)$ & $-11(3)$ & $-50(3)$ \\
\hline $\mathrm{F}(7 \mathrm{~A})$ & $88(2)$ & $170(4)$ & $117(3)$ & $-22(3)$ & $-18(2)$ & $5(2)$ \\
\hline $\mathrm{F}(8 \mathrm{~A})$ & $147(3)$ & $103(3)$ & $158(4)$ & $-9(3)$ & $42(3)$ & $34(3)$ \\
\hline $\mathrm{F}(9 \mathrm{~A})$ & $127(3)$ & $188(4)$ & $88(2)$ & $2(3)$ & $35(2)$ & $-13(3)$ \\
\hline $\mathrm{F}(10 \mathrm{~A})$ & $201(4)$ & $106(3)$ & $90(2)$ & $41(2)$ & $5(3)$ & $-32(3)$ \\
\hline $\mathrm{F}(11 \mathrm{~A})$ & $152(3)$ & $65(2)$ & $116(3)$ & $-3(2)$ & $-25(2)$ & $-22(2)$ \\
\hline $\mathrm{F}(12 \mathrm{~A})$ & $120(3)$ & $99(3)$ & $147(3)$ & $50(2)$ & $-12(3)$ & $20(2)$ \\
\hline $\mathrm{Ca}(1)$ & $47(1)$ & $47(1)$ & $48(1)$ & 0 & 0 & $1(1)$ \\
\hline
\end{tabular}




\begin{tabular}{|l|l|l|l|l|l|l|}
\hline & $\mathrm{U}_{11}$ & $\mathrm{U}_{22}$ & $\mathrm{U}_{33}$ & $\mathrm{U}_{23}$ & $\mathrm{U}_{13}$ & $\mathrm{U}_{12}$ \\
\hline $\mathrm{O}(1 \mathrm{~B})$ & $78(3)$ & $143(4)$ & $170(5)$ & $-1(3)$ & $-14(3)$ & $-20(3)$ \\
\hline $\mathrm{C}(1 \mathrm{~B})$ & $144(8)$ & $142(8)$ & $251(14)$ & $-5(7)$ & $-35(7)$ & $-48(7)$ \\
\hline $\mathrm{C}(2 \mathrm{~B})$ & $105(6)$ & $236(11)$ & $337(15)$ & $46(11)$ & $-106(8)$ & $40(7)$ \\
\hline $\mathrm{O}(2 \mathrm{~B})$ & $181(6)$ & $212(6)$ & $166(5)$ & $-104(5)$ & $88(5)$ & $-30(5)$ \\
\hline $\mathrm{C}(3 \mathrm{~B})$ & $221(11)$ & $172(9)$ & $252(12)$ & $-138(9)$ & $13(9)$ & $44(8)$ \\
\hline $\mathrm{C}(4 \mathrm{~B})$ & $301(19)$ & $710(60)$ & $70(6)$ & $-134(15)$ & $-51(11)$ & $290(30)$ \\
\hline $\mathrm{C}(5 \mathrm{~B})$ & $301(19)$ & $710(60)$ & $70(6)$ & $-134(15)$ & $-51(11)$ & $290(30)$ \\
\hline
\end{tabular}

The anisotropic displacement factor exponent takes the form: $-2 \pi^{2}\left[\mathrm{~h}^{2} \mathrm{a}^{* 2} \mathrm{U}_{11}+\ldots+2 \mathrm{~h} \mathrm{k} \mathrm{a}^{*} \mathrm{~b}^{*}\right.$ $\left.\mathrm{U}_{12}\right]$

Table S4. Bond lengths [̊] for $\mathrm{C}_{40} \mathrm{H}_{48} \mathrm{O}_{16} \mathrm{~F}_{48} \mathrm{~B}_{2} \mathrm{Ca}$ at $200 \mathrm{~K}$

\begin{tabular}{|l|r|l|r|}
\hline $\mathrm{B}(1 \mathrm{~A})-\mathrm{O}(2 \mathrm{~A})$ & $1.461(5)$ & $\mathrm{Ca}(1)-\mathrm{O}(2 \mathrm{~B}) \# 2$ & $2.433(5)$ \\
\hline $\mathrm{B}(1 \mathrm{~A})-\mathrm{O}(2 \mathrm{~A}) \# 1$ & $1.461(5)$ & $\mathrm{Ca}(1)-\mathrm{O}(2 \mathrm{~B}) \# 3$ & $2.433(5)$ \\
\hline $\mathrm{B}(1 \mathrm{~A})-\mathrm{O}(1 \mathrm{~A})$ & $1.474(5)$ & $\mathrm{Ca}(1)-\mathrm{O}(2 \mathrm{~B}) \# 4$ & $2.433(5)$ \\
\hline $\mathrm{B}(1 \mathrm{~A})-\mathrm{O}(1 \mathrm{~A}) \# 1$ & $1.474(5)$ & $\mathrm{Ca}(1)-\mathrm{O}(2 \mathrm{~B})$ & $2.433(5)$ \\
\hline $\mathrm{C}(1 \mathrm{~A})-\mathrm{O}(1 \mathrm{~A})$ & $1.384(5)$ & $\mathrm{Ca}(1)-\mathrm{O}(1 \mathrm{~B})$ & $2.476(4)$ \\
\hline $\mathrm{C}(1 \mathrm{~A})-\mathrm{C}(2 \mathrm{~A})$ & $1.507(7)$ & $\mathrm{Ca}(1)-\mathrm{O}(1 \mathrm{~B}) \# 2$ & $2.476(4)$ \\
\hline $\mathrm{C}(1 \mathrm{~A})-\mathrm{C}(3 \mathrm{~A})$ & $1.525(8)$ & $\mathrm{Ca}(1)-\mathrm{O}(1 \mathrm{~B}) \# 4$ & $2.476(4)$ \\
\hline $\mathrm{C}(1 \mathrm{~A})-\mathrm{H}(1 \mathrm{~A})$ & 1.0000 & $\mathrm{Ca}(1)-\mathrm{O}(1 \mathrm{~B}) \# 3$ & $2.476(4)$ \\
\hline $\mathrm{C}(2 \mathrm{~A})-\mathrm{F}(2 \mathrm{~A})$ & $1.308(7)$ & $\mathrm{O}(1 \mathrm{~B})-\mathrm{C}(2 \mathrm{~B})$ & $1.413(8)$ \\
\hline $\mathrm{C}(2 \mathrm{~A})-\mathrm{F}(1 \mathrm{~A})$ & $1.328(7)$ & $\mathrm{O}(1 \mathrm{~B})-\mathrm{C}(1 \mathrm{~B})$ & $1.431(8)$ \\
\hline $\mathrm{C}(2 \mathrm{~A})-\mathrm{F}(3 \mathrm{~A})$ & $1.336(7)$ & $\mathrm{C}(1 \mathrm{~B})-\mathrm{C}(1 \mathrm{~B}) \# 3$ & $1.477(15)$ \\
\hline $\mathrm{C}(3 \mathrm{~A})-\mathrm{F}(5 \mathrm{~A})$ & $1.310(8)$ & $\mathrm{C}(1 \mathrm{~B})-\mathrm{H}(1 \mathrm{BA})$ & 0.9900 \\
\hline
\end{tabular}




\begin{tabular}{|l|r|l|r|}
\hline $\mathrm{C}(3 \mathrm{~A})-\mathrm{F}(4 \mathrm{~A})$ & $1.333(7)$ & $\mathrm{C}(1 \mathrm{~B})-\mathrm{H}(1 \mathrm{BB})$ & 0.9900 \\
\hline $\mathrm{C}(3 \mathrm{~A})-\mathrm{F}(6 \mathrm{~A})$ & $1.334(8)$ & $\mathrm{C}(2 \mathrm{~B})-\mathrm{C}(5 \mathrm{~B})$ & $1.66(5)$ \\
\hline $\mathrm{C}(4 \mathrm{~A})-\mathrm{O}(2 \mathrm{~A})$ & $1.382(5)$ & $\mathrm{C}(2 \mathrm{~B})-\mathrm{H}(2 \mathrm{BA})$ & 0.9800 \\
\hline $\mathrm{C}(4 \mathrm{~A})-\mathrm{C}(5 \mathrm{~A})$ & $1.512(8)$ & $\mathrm{C}(2 \mathrm{~B})-\mathrm{H}(2 \mathrm{BB})$ & 0.9800 \\
\hline $\mathrm{C}(4 \mathrm{~A})-\mathrm{C}(6 \mathrm{~A})$ & $1.525(7)$ & $\mathrm{C}(2 \mathrm{~B})-\mathrm{H}(2 \mathrm{BC})$ & 0.9800 \\
\hline $\mathrm{C}(4 \mathrm{~A})-\mathrm{H}(4 \mathrm{~A})$ & 1.0000 & $\mathrm{O}(2 \mathrm{~B})-\mathrm{C}(4 \mathrm{~B})$ & $1.379(12)$ \\
\hline $\mathrm{C}(5 \mathrm{~A})-\mathrm{F}(7 \mathrm{~A})$ & $1.311(6)$ & $\mathrm{O}(2 \mathrm{~B})-\mathrm{C}(3 \mathrm{~B})$ & $1.462(10)$ \\
\hline $\mathrm{C}(5 \mathrm{~A})-\mathrm{F}(9 \mathrm{~A})$ & $1.328(6)$ & $\mathrm{O}(2 \mathrm{~B})-\mathrm{C}(5 \mathrm{~B}) \# 4$ & $1.88(7)$ \\
\hline $\mathrm{C}(5 \mathrm{~A})-\mathrm{F}(8 \mathrm{~A})$ & $1.330(6)$ & $\mathrm{C}(3 \mathrm{~B})-\mathrm{H}(3 \mathrm{BA})$ & 0.9800 \\
\hline $\mathrm{C}(6 \mathrm{~A})-\mathrm{F}(12 \mathrm{~A})$ & $1.309(7)$ & $\mathrm{C}(3 \mathrm{~B})-\mathrm{H}(3 \mathrm{BB})$ & 0.9800 \\
\hline $\mathrm{C}(6 \mathrm{~A})-\mathrm{F}(10 \mathrm{~A})$ & $1.326(6)$ & $\mathrm{C}(3 \mathrm{~B})-\mathrm{H}(3 \mathrm{BC})$ & 0.9800 \\
\hline $\mathrm{C}(6 \mathrm{~A})-\mathrm{F}(11 \mathrm{~A})$ & $1.326(6)$ & $\mathrm{C}(4 \mathrm{~B})-\mathrm{C}(4 \mathrm{~B}) \# 4$ & $1.49(4)$ \\
\hline & & $\mathrm{C}(4 \mathrm{~B})-\mathrm{H}(4 \mathrm{BA})$ & 0.9900 \\
\hline & & $\mathrm{C}(4 \mathrm{~B})-\mathrm{H}(4 \mathrm{BB})$ & 0.9900 \\
\hline & & $\mathrm{C}(5 \mathrm{~B})-\mathrm{H}(5 \mathrm{BA})$ & 0.9900 \\
\hline & & & 0.9900 \\
\hline
\end{tabular}

Table S5. Bond lengths $\left[\AA ̊ \cap\right.$ and angles $\left[{ }^{\circ}\right]$ for $\mathrm{C}_{40} \mathrm{H}_{48} \mathrm{O}_{16} \mathrm{~F}_{48} \mathrm{~B}_{2} \mathrm{Ca}$ at $200 \mathrm{~K}$

\begin{tabular}{|l|r|l|r|}
\hline $\mathrm{O}(2 \mathrm{~A})-\mathrm{B}(1 \mathrm{~A})-\mathrm{O}(2 \mathrm{~A}) \# 1$ & $113.6(5)$ & $\mathrm{O}(1 \mathrm{~A})-\mathrm{B}(1 \mathrm{~A})-\mathrm{O}(1 \mathrm{~A}) \# 1$ & $113.0(5)$ \\
\hline $\mathrm{O}(2 \mathrm{~A})-\mathrm{B}(1 \mathrm{~A})-\mathrm{O}(1 \mathrm{~A})$ & $108.11(16)$ & $\mathrm{O}(1 \mathrm{~A})-\mathrm{C}(1 \mathrm{~A})-\mathrm{C}(2 \mathrm{~A})$ & $108.6(5)$ \\
\hline $\mathrm{O}(2 \mathrm{~A}) \# 1-\mathrm{B}(1 \mathrm{~A})-\mathrm{O}(1 \mathrm{~A})$ & $107.06(16)$ & $\mathrm{O}(1 \mathrm{~A})-\mathrm{C}(1 \mathrm{~A})-\mathrm{C}(3 \mathrm{~A})$ & $110.4(5)$ \\
\hline
\end{tabular}




\begin{tabular}{|c|c|c|c|}
\hline $\mathrm{O}(2 \mathrm{~A})-\mathrm{B}(1 \mathrm{~A})-\mathrm{O}(1 \mathrm{~A}) \# 1$ & 107.06(16) & $C(2 A)-C(1 A)-C(3 A)$ & $112.0(5)$ \\
\hline $\mathrm{O}(2 \mathrm{~A}) \# 1-\mathrm{B}(1 \mathrm{~A})-\mathrm{O}(1 \mathrm{~A}) \# 1$ & $108.12(16)$ & $\mathrm{O}(1 \mathrm{~A})-\mathrm{C}(1 \mathrm{~A})-\mathrm{H}(1 \mathrm{~A})$ & 108.6 \\
\hline $\mathrm{C}(2 \mathrm{~A})-\mathrm{C}(1 \mathrm{~A})-\mathrm{H}(1 \mathrm{~A})$ & 108.6 & $\mathrm{O}(2 \mathrm{~A})-\mathrm{C}(4 \mathrm{~A})-\mathrm{C}(5 \mathrm{~A})$ & $108.7(4)$ \\
\hline $\mathrm{C}(3 \mathrm{~A})-\mathrm{C}(1 \mathrm{~A})-\mathrm{H}(1 \mathrm{~A})$ & 108.6 & $\mathrm{O}(2 \mathrm{~A})-\mathrm{C}(4 \mathrm{~A})-\mathrm{C}(6 \mathrm{~A})$ & $110.1(4)$ \\
\hline $\mathrm{F}(2 \mathrm{~A})-\mathrm{C}(2 \mathrm{~A})-\mathrm{F}(1 \mathrm{~A})$ & $108.4(5)$ & $C(5 A)-C(4 A)-C(6 A)$ & $113.1(5)$ \\
\hline $\mathrm{F}(2 \mathrm{~A})-\mathrm{C}(2 \mathrm{~A})-\mathrm{F}(3 \mathrm{~A})$ & $105.5(6)$ & $\mathrm{O}(2 \mathrm{~A})-\mathrm{C}(4 \mathrm{~A})-\mathrm{H}(4 \mathrm{~A})$ & 108.3 \\
\hline $\mathrm{F}(1 \mathrm{~A})-\mathrm{C}(2 \mathrm{~A})-\mathrm{F}(3 \mathrm{~A})$ & $106.9(6)$ & $\mathrm{C}(5 \mathrm{~A})-\mathrm{C}(4 \mathrm{~A})-\mathrm{H}(4 \mathrm{~A})$ & 108.3 \\
\hline $\mathrm{F}(2 \mathrm{~A})-\mathrm{C}(2 \mathrm{~A})-\mathrm{C}(1 \mathrm{~A})$ & $114.3(6)$ & $\mathrm{C}(6 \mathrm{~A})-\mathrm{C}(4 \mathrm{~A})-\mathrm{H}(4 \mathrm{~A})$ & 108.3 \\
\hline $\mathrm{F}(1 \mathrm{~A})-\mathrm{C}(2 \mathrm{~A})-\mathrm{C}(1 \mathrm{~A})$ & $112.2(6)$ & $\mathrm{F}(7 \mathrm{~A})-\mathrm{C}(5 \mathrm{~A})-\mathrm{F}(9 \mathrm{~A})$ & 107.3(5) \\
\hline $\mathrm{F}(3 \mathrm{~A})-\mathrm{C}(2 \mathrm{~A})-\mathrm{C}(1 \mathrm{~A})$ & 109.1(5) & $\mathrm{F}(7 \mathrm{~A})-\mathrm{C}(5 \mathrm{~A})-\mathrm{F}(8 \mathrm{~A})$ & $105.5(6)$ \\
\hline $\mathrm{F}(5 \mathrm{~A})-\mathrm{C}(3 \mathrm{~A})-\mathrm{F}(4 \mathrm{~A})$ & 107.4(6) & $\mathrm{F}(9 \mathrm{~A})-\mathrm{C}(5 \mathrm{~A})-\mathrm{F}(8 \mathrm{~A})$ & 107.1(5) \\
\hline $\mathrm{F}(5 \mathrm{~A})-\mathrm{C}(3 \mathrm{~A})-\mathrm{F}(6 \mathrm{~A})$ & 107.0(7) & $\mathrm{F}(7 \mathrm{~A})-\mathrm{C}(5 \mathrm{~A})-\mathrm{C}(4 \mathrm{~A})$ & $113.7(5)$ \\
\hline $\mathrm{F}(4 \mathrm{~A})-\mathrm{C}(3 \mathrm{~A})-\mathrm{F}(6 \mathrm{~A})$ & $106.5(6)$ & $\mathrm{F}(9 \mathrm{~A})-\mathrm{C}(5 \mathrm{~A})-\mathrm{C}(4 \mathrm{~A})$ & $113.0(5)$ \\
\hline $\mathrm{F}(5 \mathrm{~A})-\mathrm{C}(3 \mathrm{~A})-\mathrm{C}(1 \mathrm{~A})$ & $112.8(6)$ & $\mathrm{F}(8 \mathrm{~A})-\mathrm{C}(5 \mathrm{~A})-\mathrm{C}(4 \mathrm{~A})$ & $109.8(5)$ \\
\hline $\mathrm{F}(4 \mathrm{~A})-\mathrm{C}(3 \mathrm{~A})-\mathrm{C}(1 \mathrm{~A})$ & $112.1(6)$ & $\mathrm{F}(12 \mathrm{~A})-\mathrm{C}(6 \mathrm{~A})-\mathrm{F}(10 \mathrm{~A})$ & $107.7(5)$ \\
\hline $\mathrm{F}(6 \mathrm{~A})-\mathrm{C}(3 \mathrm{~A})-\mathrm{C}(1 \mathrm{~A})$ & $110.6(5)$ & $\mathrm{F}(12 \mathrm{~A})-\mathrm{C}(6 \mathrm{~A})-\mathrm{F}(11 \mathrm{~A})$ & $107.5(6)$ \\
\hline $\mathrm{F}(10 \mathrm{~A})-\mathrm{C}(6 \mathrm{~A})-\mathrm{F}(11 \mathrm{~A})$ & $106.5(5)$ & $\mathrm{O}(2 \mathrm{~B}) \# 3-\mathrm{Ca}(1)-\mathrm{O}(1 \mathrm{~B})$ & $77.0(2)$ \\
\hline $\mathrm{F}(12 \mathrm{~A})-\mathrm{C}(6 \mathrm{~A})-\mathrm{C}(4 \mathrm{~A})$ & $110.9(5)$ & $\mathrm{O}(2 \mathrm{~B}) \# 4-\mathrm{Ca}(1)-\mathrm{O}(1 \mathrm{~B})$ & 72.64(19) \\
\hline $\mathrm{F}(10 \mathrm{~A})-\mathrm{C}(6 \mathrm{~A})-\mathrm{C}(4 \mathrm{~A})$ & $112.2(5)$ & $\mathrm{O}(2 \mathrm{~B})-\mathrm{Ca}(1)-\mathrm{O}(1 \mathrm{~B})$ & $88.31(19)$ \\
\hline $\mathrm{F}(11 \mathrm{~A})-\mathrm{C}(6 \mathrm{~A})-\mathrm{C}(4 \mathrm{~A})$ & $111.7(5)$ & $\mathrm{O}(2 \mathrm{~B}) \# 2-\mathrm{Ca}(1)-\mathrm{O}(1 \mathrm{~B}) \# 2$ & $88.31(19)$ \\
\hline $\mathrm{C}(1 \mathrm{~A})-\mathrm{O}(1 \mathrm{~A})-\mathrm{B}(1 \mathrm{~A})$ & $121.9(3)$ & $\mathrm{O}(2 \mathrm{~B}) \# 3-\mathrm{Ca}(1)-\mathrm{O}(1 \mathrm{~B}) \# 2$ & $72.64(18)$ \\
\hline $\mathrm{C}(4 \mathrm{~A})-\mathrm{O}(2 \mathrm{~A})-\mathrm{B}(1 \mathrm{~A})$ & $122.2(3)$ & $\mathrm{O}(2 \mathrm{~B}) \# 4-\mathrm{Ca}(1)-\mathrm{O}(1 \mathrm{~B}) \# 2$ & $77.0(2)$ \\
\hline $\mathrm{O}(2 \mathrm{~B}) \# 2-\mathrm{Ca}(1)-\mathrm{O}(2 \mathrm{~B}) \# 3$ & $65.0(4)$ & $\mathrm{O}(2 \mathrm{~B})-\mathrm{Ca}(1)-\mathrm{O}(1 \mathrm{~B}) \# 2$ & $123.5(2)$ \\
\hline $\mathrm{O}(2 \mathrm{~B}) \# 2-\mathrm{Ca}(1)-\mathrm{O}(2 \mathrm{~B}) \# 4$ & $162.6(3)$ & $\mathrm{O}(1 \mathrm{~B})-\mathrm{Ca}(1)-\mathrm{O}(1 \mathrm{~B}) \# 2$ & $119.0(2)$ \\
\hline
\end{tabular}




\begin{tabular}{|l|r|l|r|}
\hline $\mathrm{O}(2 \mathrm{~B}) \# 3-\mathrm{Ca}(1)-\mathrm{O}(2 \mathrm{~B}) \# 4$ & $117.9(4)$ & $\mathrm{O}(2 \mathrm{~B}) \# 2-\mathrm{Ca}(1)-\mathrm{O}(1 \mathrm{~B}) \# 4$ & $77.0(2)$ \\
\hline $\mathrm{O}(2 \mathrm{~B}) \# 2-\mathrm{Ca}(1)-\mathrm{O}(2 \mathrm{~B})$ & $117.9(4)$ & $\mathrm{O}(2 \mathrm{~B}) \# 3-\mathrm{Ca}(1)-\mathrm{O}(1 \mathrm{~B}) \# 4$ & $123.5(2)$ \\
\hline $\mathrm{O}(2 \mathrm{~B}) \# 3-\mathrm{Ca}(1)-\mathrm{O}(2 \mathrm{~B})$ & $162.6(3)$ & $\mathrm{O}(2 \mathrm{~B}) \# 4-\mathrm{Ca}(1)-\mathrm{O}(1 \mathrm{~B}) \# 4$ & $88.31(19)$ \\
\hline $\mathrm{O}(2 \mathrm{~B}) \# 4-\mathrm{Ca}(1)-\mathrm{O}(2 \mathrm{~B})$ & $65.0(4)$ & $\mathrm{O}(2 \mathrm{~B})-\mathrm{Ca}(1)-\mathrm{O}(1 \mathrm{~B}) \# 4$ & $72.64(19)$ \\
\hline $\mathrm{O}(2 \mathrm{~B}) \# 2-\mathrm{Ca}(1)-\mathrm{O}(1 \mathrm{~B})$ & $123.5(2)$ & $\mathrm{O}(1 \mathrm{~B})-\mathrm{Ca}(1)-\mathrm{O}(1 \mathrm{~B}) \# 4$ & $157.6(2)$ \\
\hline $\mathrm{O}(1 \mathrm{~B}) \# 2-\mathrm{Ca}(1)-\mathrm{O}(1 \mathrm{~B}) \# 4$ & $65.8(2)$ & $\mathrm{O}(1 \mathrm{~B})-\mathrm{C}(2 \mathrm{~B})-\mathrm{H}(2 \mathrm{BA})$ & 109.5 \\
\hline $\mathrm{O}(2 \mathrm{~B}) \# 2-\mathrm{Ca}(1)-\mathrm{O}(1 \mathrm{~B}) \# 3$ & $72.64(18)$ & $\mathrm{C}(5 \mathrm{~B})-\mathrm{C}(2 \mathrm{~B})-\mathrm{H}(2 \mathrm{BA})$ & 84.8 \\
\hline $\mathrm{O}(2 \mathrm{~B}) \# 3-\mathrm{Ca}(1)-\mathrm{O}(1 \mathrm{~B}) \# 3$ & $88.31(19)$ & $\mathrm{O}(1 \mathrm{~B})-\mathrm{C}(2 \mathrm{~B})-\mathrm{H}(2 \mathrm{BB})$ & 109.5 \\
\hline $\mathrm{O}(2 \mathrm{~B}) \# 4-\mathrm{Ca}(1)-\mathrm{O}(1 \mathrm{~B}) \# 3$ & $123.5(2)$ & $\mathrm{C}(5 \mathrm{~B})-\mathrm{C}(2 \mathrm{~B})-\mathrm{H}(2 \mathrm{BB})$ & 151.0 \\
\hline $\mathrm{O}(2 \mathrm{~B})-\mathrm{Ca}(1)-\mathrm{O}(1 \mathrm{~B}) \# 3$ & $77.0(2)$ & $\mathrm{H}(2 \mathrm{BA})-\mathrm{C}(2 \mathrm{~B})-\mathrm{H}(2 \mathrm{BB})$ & 109.5 \\
\hline $\mathrm{O}(1 \mathrm{~B})-\mathrm{Ca}(1)-\mathrm{O}(1 \mathrm{~B}) \# 3$ & $65.8(2)$ & $\mathrm{O}(1 \mathrm{~B})-\mathrm{C}(2 \mathrm{~B})-\mathrm{H}(2 \mathrm{BC})$ & 109.5 \\
\hline $\mathrm{O}(1 \mathrm{~B}) \# 2-\mathrm{Ca}(1)-\mathrm{O}(1 \mathrm{~B}) \# 3$ & $157.6(2)$ & $\mathrm{C}(5 \mathrm{~B})-\mathrm{C}(2 \mathrm{~B})-\mathrm{H}(2 \mathrm{BC})$ & 41.6 \\
\hline $\mathrm{O}(1 \mathrm{~B}) \# 4-\mathrm{Ca}(1)-\mathrm{O}(1 \mathrm{~B}) \# 3$ & $119.0(2)$ & $\mathrm{H}(2 \mathrm{BA})-\mathrm{C}(2 \mathrm{~B})-\mathrm{H}(2 \mathrm{BC})$ & 109.5 \\
\hline $\mathrm{C}(2 \mathrm{~B})-\mathrm{O}(1 \mathrm{~B})-\mathrm{C}(1 \mathrm{~B})$ & $114.0(6)$ & $\mathrm{H}(2 \mathrm{BB})-\mathrm{C}(2 \mathrm{~B})-\mathrm{H}(2 \mathrm{BC})$ & 109.5 \\
\hline $\mathrm{C}(2 \mathrm{~B})-\mathrm{O}(1 \mathrm{~B})-\mathrm{Ca}(1)$ & $125.4(5)$ & $\mathrm{C}(4 \mathrm{~B})-\mathrm{O}(2 \mathrm{~B})-\mathrm{C}(3 \mathrm{~B})$ & $109.2(10)$ \\
\hline $\mathrm{C}(1 \mathrm{~B})-\mathrm{O}(1 \mathrm{~B})-\mathrm{Ca}(1)$ & $119.3(4)$ & $\mathrm{C}(4 \mathrm{~B})-\mathrm{O}(2 \mathrm{~B})-\mathrm{C}(5 \mathrm{~B}) \# 4$ & $93.1(16)$ \\
\hline $\mathrm{O}(1 \mathrm{~B})-\mathrm{C}(1 \mathrm{~B})-\mathrm{C}(1 \mathrm{~B}) \# 3$ & $111.9(5)$ & $\mathrm{C}(3 \mathrm{~B})-\mathrm{O}(2 \mathrm{~B})-\mathrm{C}(5 \mathrm{~B}) \# 4$ & $98(2)$ \\
\hline $\mathrm{O}(1 \mathrm{~B})-\mathrm{C}(1 \mathrm{~B})-\mathrm{H}(1 \mathrm{BA})$ & 109.2 & $\mathrm{C}(4 \mathrm{~B})-\mathrm{O}(2 \mathrm{~B})-\mathrm{Ca}(1)$ & $121.2(7)$ \\
\hline $\mathrm{C}(1 \mathrm{~B}) \# 3-\mathrm{C}(1 \mathrm{~B})-\mathrm{H}(1 \mathrm{BA})$ & 109.2 & $\mathrm{C}(3 \mathrm{~B})-\mathrm{O}(2 \mathrm{~B})-\mathrm{Ca}(1)$ & $124.1(7)$ \\
\hline $\mathrm{O}(1 \mathrm{~B})-\mathrm{C}(1 \mathrm{~B})-\mathrm{H}(1 \mathrm{BB})$ & 109.2 & $\mathrm{C}(5 \mathrm{~B}) \# 4-\mathrm{O}(2 \mathrm{~B})-\mathrm{Ca}(1)$ & $101.3(17)$ \\
\hline $\mathrm{C}(1 \mathrm{~B}) \# 3-\mathrm{C}(1 \mathrm{~B})-\mathrm{H}(1 \mathrm{BB})$ & 109.2 & $\mathrm{O}(2 \mathrm{~B})-\mathrm{C}(3 \mathrm{~B})-\mathrm{H}(3 \mathrm{BA})$ & 109.5 \\
\hline $\mathrm{H}(1 \mathrm{BA})-\mathrm{C}(1 \mathrm{~B})-\mathrm{H}(1 \mathrm{BB})$ & 107.9 & $\mathrm{O}(2 \mathrm{~B})-\mathrm{C}(3 \mathrm{~B})-\mathrm{H}(3 \mathrm{BB})$ & 109.5 \\
\hline $\mathrm{O}(1 \mathrm{~B})-\mathrm{C}(2 \mathrm{~B})-\mathrm{C}(5 \mathrm{~B})$ & $\mathrm{H}(4 \mathrm{BA})-\mathrm{C}(4 \mathrm{~B})-\mathrm{H}(4 \mathrm{BB})$ & 109.3 \\
\hline $\mathrm{H}(3 \mathrm{BA})-\mathrm{C}(3 \mathrm{~B})-\mathrm{H}(3 \mathrm{BB})$ & & & \\
\hline
\end{tabular}




\begin{tabular}{|l|r|l|r|}
\hline $\mathrm{O}(2 \mathrm{~B})-\mathrm{C}(3 \mathrm{~B})-\mathrm{H}(3 \mathrm{BC})$ & 109.5 & $\mathrm{C}(2 \mathrm{~B})-\mathrm{C}(5 \mathrm{~B})-\mathrm{O}(2 \mathrm{~B}) \# 4$ & $116(2)$ \\
\hline $\mathrm{H}(3 \mathrm{BA})-\mathrm{C}(3 \mathrm{~B})-\mathrm{H}(3 \mathrm{BC})$ & 109.5 & $\mathrm{C}(2 \mathrm{~B})-\mathrm{C}(5 \mathrm{~B})-\mathrm{H}(5 \mathrm{BA})$ & 108.3 \\
\hline $\mathrm{H}(3 \mathrm{BB})-\mathrm{C}(3 \mathrm{~B})-\mathrm{H}(3 \mathrm{BC})$ & 109.5 & $\mathrm{O}(2 \mathrm{~B}) \# 4-\mathrm{C}(5 \mathrm{~B})-\mathrm{H}(5 \mathrm{BA})$ & 108.3 \\
\hline $\mathrm{O}(2 \mathrm{~B})-\mathrm{C}(4 \mathrm{~B})-\mathrm{C}(4 \mathrm{~B}) \# 4$ & $111.4(15)$ & $\mathrm{C}(2 \mathrm{~B})-\mathrm{C}(5 \mathrm{~B})-\mathrm{H}(5 \mathrm{BB})$ & 108.3 \\
\hline $\mathrm{O}(2 \mathrm{~B})-\mathrm{C}(4 \mathrm{~B})-\mathrm{H}(4 \mathrm{BA})$ & 109.4 & $\mathrm{O}(2 \mathrm{~B}) \# 4-\mathrm{C}(5 \mathrm{~B})-\mathrm{H}(5 \mathrm{BB})$ & 108.3 \\
\hline $\mathrm{C}(4 \mathrm{~B}) \# 4-\mathrm{C}(4 \mathrm{~B})-\mathrm{H}(4 \mathrm{BA})$ & 109.4 & $\mathrm{H}(5 \mathrm{BA})-\mathrm{C}(5 \mathrm{~B})-\mathrm{H}(5 \mathrm{BB})$ & 107.4 \\
\hline $\mathrm{O}(2 \mathrm{~B})-\mathrm{C}(4 \mathrm{~B})-\mathrm{H}(4 \mathrm{BB})$ & 109.3 & & \\
\hline
\end{tabular}

Symmetry transformations used to generate equivalent atoms:

$\# 1-x+1 / 2, y,-z+1 \quad \# 2-y+3 / 4,-x+3 / 4,-z+3 / 4 \quad \# 3$ y-3/4,x+3/4,-z+3/4 \#4 -x+0,-y+3/2,z+0

\section{SI.5 References:}

(1) Fischer, L. M.; Tenje, M.; Heiskanen, A. R.; Masuda, N.; Castillo, J.; Bentien, A.; Émneus, J.; Jakobsen, M. H.; Boisen, A., Gold cleaning methods for electrochemical detection applications. Microelectron. Eng. 2009, 86 (4-6), 1282-1285.

(2) Sun, X.; Lee, H.; Yang, X.; McBreen, J., A new additive for lithium battery electrolytes based on an alkyl borate compound. J. Electrochem. Soc. 2002, 149 (3), A355-A359. 